\title{
Correlation Functions for Quasi-Linear Response Theory
}

\author{
W. Brenig \\ Physik-Department, Technische Universität München, D-8046 Garching, Federal Republic of \\ Germany
}

\begin{abstract}
A "quasi-linear" regression formula is derived by an expansion around quasi-static equilibrium. It relates the relaxation of thermodynamic "forces" to the regression of correlations of thermodynamic "coordinates" in quasi-static equilibrium. Correlation functions and memory kernels can be introduced in almost complete analogy to linear response theory. A non-linear, non-Markovian kinetic equation is derived. The kinetic coefficients are given in terms of correlation functions of stochastic forces in quasi-static equilibrium similar to the linear theory.
\end{abstract}

\section{Introduction}

Since the historical work of Langevin (1908) [1] on Brownian motion a vast amount of literature on nonequilibrium statistical mechanics has accumulated in which kinetic coefficients are expressed as limiting values of correlation functions. Let us mention only a small selection of important papers [2-7] and a few review papers and books [8-11] for further references. The aim of nonequilibrium statistical mechanics in general is to study the evolution in time of expectation values

$$
Q_{k}(t)=\overline{q_{k}(t)}=\operatorname{trace}\left(q_{k}(t) \varrho(0)\right)
$$

of a set of operators $q_{k}(t)$ in an ensemble described by the statistical operator $\varrho(0)$. We are going to use the Heisenberg picture throughout with the equations of motion

$$
d q_{k}(t) / d t=\frac{i}{\hbar}\left[H, q_{k}(t)\right]
$$

The evaluation of the right hand side of (1) for $q_{k}(t)$ a solution of (2) so far has only been possible in case $\varrho(0)$ is sufficiently close to equilibrium. Usually one considers ensembles in "quasi-static" equilibrium described by the operators

where

$$
\varrho=\exp [-\beta(I(t)-K(t))]
$$

$$
I(t)=H-\sum_{k} q_{k}^{*}(t) f_{k}(t)
$$


is the enthalpy operator and $K(t)$ the Gibbs free enthalpy. $q_{k}^{*}$ is the hermitian conjugate of $q_{k}$ and $I(t)=I^{*}(t)$ is supposed to be hermitian. $\varrho$ according to (3) describes a state of maximum entropy under the subsidiary conditions that $\operatorname{trace}\left(q_{k}(t) \varrho\right)=\left\langle q_{k}(t)\right\rangle$ and the total energy trace $(H \varrho)=\langle H\rangle$ have certain given values. We are going to choose the Lagrange multipliers $\beta$ and $f_{k}(t)$ so that $\left\langle q_{k}(t)\right\rangle=Q_{k}(t)$ and $\langle H\rangle=E$. The initial condition most widely used $[5,8,10]$ assumes that at time $t=0$ the ensemble is prepared in quasistatic equilibrium (for instance by external manipulations)

$$
\varrho(0)=\exp [-\beta(I(0)-K(0))],
$$

and from thereon evolves according to its own internal dynamics (1) and (2).

Even for the simple initial condition (5) the right hand side of (1) in general can not be expressed in terms of tractable quantities. Two further approximations have been considered: The deviations $Q_{k}(t)$ from their equilibrium values are assumed to be either small or slowly varying. In the first case the right hand side of (1) is expanded in powers of $f_{k}$. To first order in $f_{k}$ one then obtains the so-called linear response theory which forms the basis of a large part of modern nonequilibrium theory (compare for instance $[5,6,8-10]$ ). In the second case one can expect at all times (not only at $t=0$ ) the ensemble to be close to quasi-static equilibrium, i.e. $\varrho(0)$ can approximately be replaced by $\varrho$ according to (3). The equations of motion for $Q_{k}(t)$ then are approximately Markovian.

A microscopic basis for both cases can be obtained by a suitable expansion of $\varrho(0)$ in terms of $\varrho$. Such an expansion around quasi-static equilibrium has been attempted frequently in the literature but the results in most cases have been less tractable than the corresponding ones of linear response theory. Perhaps the most advanced technique in this respect has been proposed in [7]. It has in fact initiated the investigations presented in this paper.

In contrast to [7] we use the initial condition (5) in order to have a direct and straightforward connection to linear response theory $[5,6]$. We then derive a quasi-linear regression formula which relates the relaxation of the Lagrange parameters to the regression of correlations in quasi-static equilibrium. The retarded correlation functions then can be handled in almost complete analogy to the linear theory. In particular, memory kernels can be introduced similar to linear theory. The time integrals of these memory kernels govern the low frequency (long-time) behavior of the correlation functions and can be identified as generalized kinetic coefficients. This way a nonlinear generalization of Onsager's nonequilibrium theory is obtained. On the other hand, expanding the quasi-static equilibrium around the static equilibrium, one recovers the results of linear response theory including all memory effects of this theory.

\section{A "Quasi-Linear" Regression Formula}

The starting point of quasi-linear response theory is an expansion of $\varrho(0)$ in (5) to first order in the quantity

$$
\Delta(t)=\Delta I(t)-\Delta I(0)=\sum\left[\Delta q_{k}^{*}(t) f_{k}(t)-\Delta q_{k}^{*}(0) f_{k}(0)\right] .
$$


We indicate averages with $\varrho(0)$ by a bar $(-)$ and (quasi-static) averages with $\varrho$ by brackets $(\langle\rangle)$ and use the notation $\Delta A=A-\langle A\rangle$ for the deviation of operators from their quasi-static equilibrium values. The expansion of $\varrho(0)$ then reads

$$
\varrho(0)=\left(1+\int_{0}^{\beta} e^{-\alpha I(t)} \Delta(t) e^{\alpha I(t)} d \alpha+\ldots\right) \varrho .
$$

This expansion now can be used to calculate the difference $\overline{\Delta A}$ between exact and quasi-static averages to first order in $\Delta(t)$. One finds

$$
\overline{\Delta A}=\beta\langle\Delta A ; \Delta(t)\rangle,
$$

where we have used the notation

$$
\beta\langle A ; B\rangle=\int_{0}^{\beta}\left\langle A e^{-\alpha I(t)} B^{*} e^{\alpha I(t)}\right\rangle d \alpha .
$$

The quantities introduced this way have the symmetry, linearity and positivity properties of a scalar product $[5,6]$ with, for instance, $\langle A ; A\rangle \geqq 0$, a Schwartz inequality and all that. (8) becomes particularly simple if $A$ is taken to be one of the operators $q_{k}(t)$. Since the $f_{k}(t)$ are chosen so as to make the quasi-static averages $\left\langle q_{k}(t)\right\rangle$ equal to the exact averages $Q_{k}(t)$, the left hand side of (8) vanishes:

$$
0=\left\langle\Delta q_{k}(t) ; \Delta(t)\right\rangle .
$$

Inserting the right hand side of (6) leads to

$$
\sum_{l}\left\langle\Delta q_{k}(t) ; \Delta q_{l}(t)\right\rangle f_{l}(t)=\sum_{l}\left\langle\Delta q_{k}(t) ; \Delta q_{l}(0)\right\rangle f_{l}(0) .
$$

This formula relates the relaxation of $f_{k}(t)$ to the regression of correlations of the $q_{k}(t)$ with their initial values $q_{k}(0)$ in quasi-static equilibrium. (11) is only apparently linear in $f_{k}(t)$ since the expectation values on both sides of (11) depend on the $f_{k}(t)$ via (3).

Equation (11) obviously becomes exact for $t \rightarrow 0$ independent of the magnitude of $f_{k}(0)$. It also becomes exact in the linear regime when the expectation values on both sides of (11) can be replaced by equilibrium expectation values, replacing $I(t)$ in (3) and (9) by $H$. Then using

$$
Q_{k}(t)=\sum_{l} \beta\left\langle\Delta q_{k}(t) ; \Delta q_{l}(t)\right\rangle_{0} f_{l}(t),
$$

which is valid in the linear regime $\left(\langle\rangle_{0}\right.$ indicating equilibrium expectation values) (11) becomes identical to the regression formula

$$
Q_{k}(t)=\sum_{l} \beta\left\langle\Delta q_{k}(t) ; \Delta q_{l}(0)\right\rangle_{0} f_{l}(0)
$$

of linear response theory [5].

For arbitrary times and arbitrary magnitude of $f_{k}(0)(11)$ can only be expected to be valid if the system is sufficiently close to quasi-static equilibrium, more precisely, if variables different from the $q_{k}$ (in particular their time derivatives $\dot{q}_{k}$ ) which are initially not in quasi-static equilibrium relax to their quasi-static equilibrium values sufficiently fast. Let, for instance, $\tau_{\text {micro }}$ be the corresponding 
(so-called "microscopic") relaxation time then (11) can be expected to be valid for $t \gg \tau_{\text {micro }}$. Of course (11) then is only interesting, if the (so-called "macroscopic") relaxation time $\tau_{\text {macro }}$ of the $f_{k}(t)$ themselves is sufficiently large compared to $\tau_{\text {micro. }}$. Otherwise (11) would only be valid when the $f_{k}(t)$ have relaxed to their equilibrium values which (for ergodic systems) are $f_{k}(\infty)=0$.

The discussion so far may suffice to indicate that (11) can be a basis for a theory which contains the nonlinearities of systems far away from equilibrium as well as the short time (high frequency) and memory effects which are known from linear response theory.

\section{Retarded Correlation Functions and Memory Kernels}

In this section we are going to demonstrate that apart from a few additional precautions, the theory of correlation functions in the linear regime can be directly generalized to the quasi-linear regime. The main aim of that theory is (i) to cast the correlation functions into a form from which their behavior at small times $t \ll \tau_{\text {micro }}$ as well as at large times $t \gg \tau_{\text {micro }}$ can be easily read off and be parametrized in terms of certain numbers (sum rule values, kinetic coefficients) and (ii) to derive "microscopic" expressions for these numbers which are convenient for practical calculation.

For times $t \geqq 0$ it is sufficient to consider retarded functions. In order to have a quantity more symmetric in initial and final times than the right hand side of (11) we replace the time $t$ in (3) by some fixed value $t_{0}$ anywhere between $t$ and 0 . We also replaced the initial time $t=0$ by some arbitrary value $t^{\prime}$ and define the relaxation function

$$
\phi_{k l}\left(t, t^{\prime}\right)=i \beta\left\langle\Delta q_{k}(t) ; \Delta q_{l}\left(t^{\prime}\right)\right\rangle \theta\left(t-t^{\prime}\right)
$$

in analogy to the linear case [5]. The averages, however, are taken with a quasistatic equilibrium ensemble (3) at some time $t_{0}$. The correlation functions occurring at the right hand side of (11) are obtained from (14) by putting $t_{0}=t$ and $t^{\prime}=0$. In order to simplify some of the formal manipulations we introduce a generalized matrix notation with

$$
(A B)_{k m}\left(t, t^{\prime}\right)=\sum_{l} \int_{-\infty}^{\infty} A_{k l}\left(t, t^{\prime \prime}\right) B_{l m}\left(t^{\prime \prime}, t^{\prime}\right) d t^{\prime \prime}
$$

and define three matrices: The quasi-static (isothermal) susceptibility

$$
\chi_{k l}^{T}\left(t, t^{\prime}\right)=\beta\left\langle\Delta q_{k}(t) ; \Delta q_{l}(t)\right\rangle \delta\left(t-t^{\prime}\right),
$$

the differentiation with respect to time

$$
z_{k l}\left(t, t^{\prime}\right)=i \delta_{k l} d \delta\left(t-t^{\prime}\right) / d t
$$

and the socalled memory kernel $R$ by

$$
\phi=\chi^{T}(R-z)^{-1} .
$$

It will turn out that $R$ is of the form

$$
R_{k l}\left(t, t^{\prime}\right)=R_{k l}^{0}(t) \delta\left(t-t^{\prime}\right)+R_{k l}^{1}\left(t, t^{\prime}\right) \theta\left(t-t^{\prime}\right) .
$$


We now put $t_{0}=t, t^{\prime}=0$ and make use of (11), (18), (19) and the exact relation

to obtain

$$
\dot{Q}_{k}(t)=\sum_{l} \chi_{k l}^{T}(t) \dot{f}_{l}(t)
$$

$$
\dot{Q}_{k}(t)=-i \sum_{l} \int_{0}^{t+0}\left(\chi^{T} R\right)_{k l}\left(t, t^{\prime}\right) f_{l}\left(t^{\prime}\right) d t^{\prime} .
$$

We have added the positive infinitesimal +0 to the upper limit of the integral in order not to lose the singular part $R^{0}$ of (19). (21) is the kinetic equation, nonlinear and non-Markovian, but of the same structure as in the linear Markovian regime. The matrix elements of $\left(\chi^{T} R\right)$ may be considered as generalized kinetik coefficients. Next we multiply (18) by $z$ from the left and by $(R-z)$ from the right. Then using (14) one finds after some rearrangements [with $\Delta q_{k}(t)=q_{k}(t)-Q_{k}(t)$ and $\Delta \dot{q}_{k}(t)$ $\left.=\dot{q}_{k}(t)-\dot{Q}_{k}(t)\right]$

$$
\begin{aligned}
\left(\chi^{T} R\right)_{k l}\left(t, t^{\prime}\right)= & -i \beta\left\langle\Delta q_{k}(t) ; \Delta \dot{q}_{l}(t)\right\rangle \delta\left(t-t^{\prime}\right)-i \beta\left\langle\Delta \dot{q}_{k}(t) ; \Delta \dot{q}_{l}\left(t^{\prime}\right)\right\rangle \theta\left(t-t^{\prime}\right) \\
& +\sum \beta \int\left\langle\Delta \dot{q}_{k}(t) ; \Delta q_{m}\left(t^{\prime \prime}\right)\right\rangle \theta\left(t-t^{\prime \prime}\right) R_{m l}\left(t^{\prime \prime}, t^{\prime}\right) d t^{\prime \prime} .
\end{aligned}
$$

From this one can identify $R^{0}$ as [compare (19)]

$$
\left(\chi^{T} R^{0}\right)_{k l}(t)=-i \beta\left\langle\Delta q_{k}(t) ; \Delta \dot{q}_{l}(t)\right\rangle .
$$

For the remainder of $R$ (22) yields an inhomogeneous linear integral equation. The von Neumann series of this equation term by term is proportional to $\theta\left(t-t^{\prime}\right)$ which proves (19). Equations (18) and (22) then can be written in a more transparent form by introducing the quantity

$$
F_{l}\left(t^{\prime}\right)=\Delta \dot{q}_{l}\left(t^{\prime}\right)-i \sum_{m} \int_{t^{\prime}}^{t} \Delta q_{m}\left(t^{\prime \prime}\right) R_{m l}^{*}\left(t^{\prime \prime}, t^{\prime}\right) d t^{\prime \prime} \quad \text { for } \quad t \geqq t^{\prime} .
$$

Then, first of all; one has

$$
\overline{F_{l}\left(t^{\prime}\right)}=0 ; \quad t \geqq t^{\prime}
$$

Secondly, multiplying $(18)$ by $(R-z)$ from the right one finds after some rearrangements

$$
\left\langle\Delta q_{k}(t) ; F_{l}\left(t^{\prime}\right)\right\rangle \theta\left(t-t^{\prime}\right)=0,
$$

and thirdly (22) can be rewritten as

$$
\left(\chi^{T} R\right)_{k l}\left(t, t^{\prime}\right)=-i \beta\left\langle\Delta q_{k}(t) ; \Delta \dot{q}_{l}(t)\right\rangle \delta\left(t-t^{\prime}\right)-i \beta\left\langle\Delta \dot{q}_{k}(t) ; F_{l}\left(t^{\prime}\right)\right\rangle \theta\left(t-t^{\prime}\right) .
$$

Special approximate versions of the Eq. (25)-(27) appear already in Langevin's [1] paper on Brownian motion, where $F$ is the so-called "stochastic force." $F$ has zero average, zero correlation with the $\Delta q$ whereas the correlations with $\Delta \dot{q}$ yield the kinetic coefficients via the generalized Langevin-Kubo formula. In the Langevin treatment and in its generalizations according to linear response theory the stochastic forces are defined so as to make (25) identical with the kinetic equation (21). In our nonlinear generalization (25) is a trivial consequence of the occurrence of the deviations $\Delta q$ and $\Delta \dot{q}$ from the averages $Q$ and $\dot{Q}$ in (24). By a slightly more elaborate definition than (24) one could make (25) identical with (21). Since, 
however we have a simple derivation of (21) from (18) and (11) we refrain from such a definition. The main difference between the nonlinear kinetic coefficients (27) and the ones given in $[7,9]$ is the occurrence of the second term on the right hand side of (24). This subtraction term proportional to $R$ eliminates slowly decaying correlations of the "velocities" $\dot{q}(t)$ from the kinetic coefficients, an effect which is known from the linear regime, and taken into account already in Langevin's treatment (although in some cases [12] it may not be necessary).

\section{The Increase of Entropy}

In linear response theory the "propagation" of Heisenberg operators in $t$ as well as statistical operators in $\beta$ both are governed by the same operator $H$. As a consequence the correlation functions obey the Kubo [5] symmetry relations closely related to the detailed balance condition [4]. From this condition certain positivity properties can be derived. For instance the spectral functions of correlation function matrices are positive matrices and the matrix of the kinetic coefficients, too, has a positive spectral function. This in turn is closely related to the positivity of the rate of change of entropy $S(t)=-\langle\ln \varrho\rangle$ for a closed system

$$
d S(t) / d t=-\beta \sum f_{k}^{*}(t) d Q_{k}(t) / d t
$$

In quasi-linear theory the statistical operators (3), (5) propagate with the enthalpy $I$ rather than the energy $H$. Thus detailed balance does not hold. Arguments for the positivity of the right hand side of (28) invoking detailed balance (compare for instance [9, p. 334]) are not valid. However, one still has the rigorous validity of the inequality

$$
\overline{\ln \varrho(0)} \geqq \overline{\ln \varrho}=\langle\ln \varrho\rangle,
$$

which implies $S(t) \geqq S(0)$. Integrating the (rigorous) equation (28) from 0 to $t$ one finds using (21) the inequality

$$
i \sum_{k, l} \int_{0}^{t+0} d t^{\prime} d t^{\prime \prime} f_{k}\left(t^{\prime}\right)\left(\chi^{T} R\right)\left(t^{\prime}, t^{\prime \prime}\right) f_{l}\left(t^{\prime \prime}\right) \geqq 0 .
$$

Thus, at least this special "average" of the matrix of kinetic coefficients is positive. Regarding the right hand side of (28) and the left hand side of (30), it may be interesting to note that the singular part $R^{0}$ of $R$ according to (23) contributes zero in both cases and thus corresponds to the "reversible" part of $\dot{Q}_{k}(t)$ just as in the linear regime. This follows from

$$
\beta \sum_{l}\left\langle A ; \dot{q}_{l}(t)\right\rangle f_{l}(t)=\frac{i}{\hbar} \sum_{l}\left\langle\left[A, q_{l}^{*}(t)\right]\right\rangle f_{l}(t),
$$

which can easily be verified for any operator $A$ from (2) and (3). Now using (21), (23), (28), and (31) one obtains

$$
\begin{aligned}
(d S(t) / d t)^{0} & =-\beta \sum_{k, l} f_{k}^{*}(t) R_{k l}^{0}(t) f_{l}(t) \\
& =\frac{i}{\hbar}\left\langle\left[\sum_{k} f_{k}^{*}(t) q_{k}(t), \sum_{l} f_{l}(t) q_{l}^{*}(t)\right]\right\rangle=0,
\end{aligned}
$$


since the two sums in the commutator are hermitian and thus identical. It may also be interesting to mention that for $t=0$ the contribution of the regular part $R^{1}$ to (21) vanishes. Thus

$$
\dot{Q}_{k}(0)=\frac{i}{\hbar} \sum_{l}\left\langle\left[q_{k}(0), q_{l}^{*}(0)\right]\right\rangle f_{l}(0) .
$$

On the other hand one has the rigorous equation of motion

$$
\dot{Q}_{k}(t)=\frac{i}{\hbar} \sum_{l} \overline{\left[q_{k}(t), q_{l}^{*}(0)\right]} f_{l}(0)
$$

following from (2) and (5) which agrees exactly with (33) at $t=0$. Our regression formula (11) leading to (33) therefore not only reproduces the exact value of $Q_{k}(0)$ but also of $\dot{Q}_{k}(0)$.

\section{References}

1. Langevin, P.: C.R. Paris 146, 530 (1908)

2. Nyquist, H.: Phys. Rev. 32, 110 (1928)

3. Onsager, L.: Phys. Rev. 37, 405 (1931) Onsager, L.: Phys. Rev. 38, 2265 (1931)

4. Callen, H.B., Welton, T.A.: Phys. Rev. 83, 34 (1951)

5. Kubo, R.: J. Phys. Soc. (Japan) 12, 570 (1957)

6. Mori, H.: Progr. Theor. Phys. 34, 399 (1965)

7. Zubarev, D.N.: Dokl. Akad. Nauk. SSSR 140, 92 (1961) [Sov. Phys.-Dokl. 6, 776 (1962)]

8. Kubo, R.: In: Lectures in Theoretical Physics, Vol. I, W.E. Brittin, L.G.Dunham (eds.), p. 120. New York: Interscience 1959

9. Zubarev, D.N.: Nonequilibrium statistical mechanics. Moscow: Nauka Press 1971 (Engl. transl. New York: Consultants Bureau 1974)

10. Martin, P.C.: In: Statistical mechanics of equilibrium and nonequilibrium, Meixner, J. (ed.), p. 100. Amsterdam: North-Holland 1969

11. Forster, D.: Hydrodynamic fluctuations, broken symmetry and correlation functions. New York: Benjamin 1975

12. Berne, B.J., Boon, J.P., Rice, S.A. : J. Chem. Phys. 45, 1086 (1966)

Communicated by R. Jost

Received September 15, 1981 
